\title{
Proteasom-Inhibitor nun auch subkutan
}

\author{
Der Proteasom-Inhibitor Bortezomib \\ kann nun auch subkutan verabreicht \\ werden. Die Applikation ist ebenso \\ wirksam wie die intravenöse Gabe, \\ jedoch besser verträglich.
}

Bortezomib (Velcade ${ }^{\star}$ ) ist etablierter Bestandteil in der Therapie des multiplen Myeloms. Hans-Jürgen Salwender, Hamburg, erklärte, dass eine Bortezomib-Therapie die Zeit bis zum Krankheitsprogress in der Primär- sowie der Rezidivtherapie deutlich verlängern und das Mortalitätsrisiko senken kann.

Bislang war der Proteasom-Inhibitor nur für die intravenöse (i.v.) Gabe zugelassen. „Eine unerwünschte Nebenwirkung, die bei dieser Applikationsweise gehäuft auftritt, sind periphere Neuropathien", betonte Hartmut Goldschmidt, Heidelberg. Dem konnte bislang nur durch Dosisanpassungen bis hin zum Therapieabbruch begegnet werden. In einer Nichtunterlegenheitsstudie bei Patienten mit rezidiviertem multiplen Myelom erwies sich die subkutane Gabe als besser verträgliche Alternative zur intravenösen [Moreau P et al. Lancet Oncol. 2011;12(5):431-40]. Besonders periphere Neuropathien aller Schweregrade traten mit 38 vs. $53 \%$ seltener auf als nach i.v.-Applikation. Dabei unterschieden sich das Ansprechen auf die Behandlung, die Rate der kompletten und partiellen Remissionen, das progressionsfreie sowie das Gesamtüberleben in den beiden Studienarmen nicht signfikant. Zudem ist die Behandlung laut Goldschmidt einfacher - auch Patienten mit schlechtem Venenzugang können gut behandelt werden. Silke Wedekind

Pressekonferenz „Bessere Verträglichkeit bei bewährter Wirksamkeit - Velcade ${ }^{\circledast}$ s. c. zur Therapie des multiplen Myeloms", Frankfurt/Main, 25. September 2012; Veranstalter: Janssen-Cilag

\section{Personalisierte Therapie bei nicht resektabler Erkrankung}

\section{Jeder zweite Patient mit metastasier- tem kolorektalen Karzinom (mCRC) erhält heute eine Drittlinientherapie. Dabei bestimmt die Wahl der ersten Behandlungsoption die folgenden Möglichkeiten.}

Bei einem metastasierten kolorektalen Karzinom (mCRC) mit KRAS-Wildtyp kann eine intensive Therapie mit Cetuximab (Erbitux ${ }^{\oplus}$ ) kombiniert mit einer Standardchemotherapie vorteilhaft sein. Im Rahmen mehrerer Studien profitierten Patienten mit mCRC und einem KRASWildtyp von Cetuximab zusätzlich zur Standard-Erstlinientherapie: Die gepoolte Analyse der Studien CRYSTAL und OPUS mit 845 ausgewerteten Patienten zeigte ein signifikant längeres Gesamtüberleben unter dieser Kombination [Bokemeyer C et al. Eur J Cancer. 2012;48(10):1466-75]. Der BRAF-Mutationsstatus war kein prädiktiver Marker für das Therapieansprechen, ging aber mit einer schlechteren Prognose einher. Auch über 70-jährige Patienten profitierten von dieser Kombination. Das mediane Gesamtüberleben war in der gepoolten Auswertung für diese Subgruppe mit dem jüngerer Patienten vergleichbar (23,3 vs. 23,6 Monate) und deutlich höher als bei den über 70-Jährigen, die nur eine Standardchemotherapie erhalten hatten $(15,1$ Monate). Cetuximab ist nach Claus-Henning Köhne, Oldenburg, ein Standard in der Erstlinientherapie für Patienten mit mCRC bei KRAS-Wildtyp - auch für ältere asymptomatische Patienten mit nicht resektablen Metastasen. Friederike Klein

Satellitensymposium „ERBITUX in der palliativen Erstlinientherapie des mCRC" anlässlich der Jahrestagung der Deutschen, Österreichischen und Schweizerischen Gesellschaften für Hämatologie und Onkologie, Stuttgart, 19. Oktober 2012; Veranstalter: Merck Serono

\section{NMH Standard bei Risikopatienten}

Zur Thromboseprophylaxe ist bei $\mathrm{Pa}$ tienten mit Risikofaktoren wie Immobilisierung oder Tumoroperation die Gabe von niedermolekularen Heparinen (NMH) Therapiestandard. Nach Gabriele Faerber, Hamburg, führen jedoch nur $5 \%$ der Onkologen eine routinemäßige Thromboseprophylaxe durch. Tumorpatienten mit einer Thrombose sollten anstelle von Vitamin-K-Antagonisten für drei bis sechs Monate mit NMH behandelt werden. Dabei sind Substanzen wie Tinzaparin (Innohep ${ }^{\oplus}$ ) mit hohem Molekulargewicht und geringer $\mathrm{Ku}$ mulation der Anti-Xa-Aktivität zu bevorzugen.

Nach Informationen von Leo

\section{Sandler-Schema bei NSCLC bestätigt}

Im Vergleich zum Sandler-Schema mit Carboplatin/Paclitaxel plus Bevacizumab (Avastin ${ }^{\circledast}$ ) führt der Ersatz des Taxans durch Pemetrexed zu keinem Überlebensvorteil. Dies geht aus einer Studie hervor, die kürzlich in Chicago vorgestellt wurde [Patel J et al. IASLC 2012;Abstract \#LBA553]. In der Untersuchung erhielten 939 Patienten mit nicht-kleinzelligem Lungenkarzinom (NSCLC) ohne Plattenepithel-Histologie als First-Line-Therapie im experimentellen Arm Carboplatin/Pemetrexed plus Bevacizumab $(\mathrm{n}=472)$. Im Kontrollarm $(\mathrm{n}=467)$ verabreichten die Ärzte das SandlerSchema mit Carboplatin/Paclitaxel plus Bevacizumab. Bei Patienten ohne Krankheitsprogression erfolgte anschließend eine Erhaltungstherapie, wobei die Patienten im experimentellen Arm die Kombination aus Pemetrexed und Bevacizumab erhielten, während im Kontrollarm der VEGFAntikörper zulassungsgemäß als Monotherapie gegeben wurde. Das mediane Gesamtüberleben war unter dem aufwendigeren Pemetrexed-Regime nicht länger als beim Sandler-Schema $(12,6$ vs. 13,4 Monate; $\mathrm{p}=0,949)$.

Nach Informationen von Roche 